\title{
The Universe, a Personal View on Exploring the Boundaries, or: The Science of the Extremes*
}

\author{
Gerard 't Hooft'
}

What are the fundamental equations of motion when we consider objects that move faster, have tinier structures, higher mass densities, higher or lower temperatures than ever studied before? Here, we emphasize the importance of studying extreme conditions and situations in science so as to uncover the basic laws of nature. One of the important extremes is extreme complexity, a necessary condition for the emergence of life.

Keywords: Extremely Fast; Extremely Tiny; Extremely Dense; Extremely Heavy; Relativity Quantum; Space-Time; Gravitation.

This account is a thorough rewriting of a recording of a talk I gave with this title in the Swedish Academy of Sciences, where I happen to have left many pleasant memories. Most other talks I attended at this meeting, were about life sciences, which, due to their severe complexity, are difficult to fathom by a pure sang theoretical physicist such as myself. It so happens however, that some of our interests do coincide. One question sometimes posed by biologists concerns the origin of left-right asymmetry in living organisms. Actually, the history of the evolution of life forms on planet Earth shows that living organisms began by having much more symmetry: they may have started out being practically spherical. Then, when organisms needed a head and a tail, spherical symmetry was reduced to cylindrical symmetry. Subsequently, living organisms such as snails, discovered that structures become sturdier when you curl them up. These organisms, such as the ammonites, were left with only left-right symmetry. Finally, most modern snails also break that symmetry, which again seems to have improved the sturdiness of the structure of their shells.

Here, the question is whether and how a competition between life forms and their mirror images may have emerged, with the predictable outcome that one of the two won. Most snails seem to be twisted in the same "right-handed" direction. Why not left-handed? Whence this asymmetry? Some researchers suspect that the origin of this asymmetry can be traced back to the minute mirror asymmetries that were found to exist in the fundamental laws of Nature. For physicists such as me, however, a simpler answer to the asymmetry question seems to be obvious. All these symmetries probably broke spontaneously, as we often see happen even in non-living materials, such as magnetic material, and even sub-atomic particles. No external asymmetric force is needed at all for symmetries to get broken. Note that our environment breaks left-right symmetry in a big way, an asymmetry that cannot be directly attributed to asymmetries in the fundamental laws of physics.

For the life sciences, life in extreme conditions is often interesting. How does life endure extreme cold, extreme heat, extreme acidity, the extremely high pressures of the deepest trenches in the oceans, and so on? As a scientist, I am particularly interested in the conditions on the other planets and asteroids in our solar system. Being extreme in many ways,

\footnotetext{
${ }^{1}$ Institute for Theoretical Physics, Postbox 80.089, 3508 TB Utrecht. The Netherlands. Published online 16 May 2017; doi:10.1142/ S2529732517400041

*This article was transcribed from a presentation delivered by Professor Gerard 't Hooft at the Molecular Frontiers Symposium, Royal Swedish Academy of Sciences, May 2013. It is published here in MFJ with permission from Professor Gerard 't Hooft.

This is an Open Access article published by World Scientific Publishing Company. It is distributed under the terms of the Creative Commons Attribution 4.0 (CC-BY) License. Further distribution of this work is permitted, provided the original work is properly cited.
} 
they form interesting challenges for human life when we contemplate the possibility of establishing human colonies there. The conceivable possibility to establish a human colony on planet Mars is seriously being considered today, not only by agencies such as NASA, but also by private groups, such as "Mars One", established by some compatriots of mine. This however can better be left for an other talk.

From a physics point of view, the conditions just mentioned are not extreme at all. Even the center of the Earth, and the Sun, are not yet overly extreme from a physical point of view. We know of planets, stars, and regions in the distant or the early universe, where conditions are/were far more extreme. My main subject today is the extremes in physics. We can extrapolate the laws of non-living matter very well in most places, but there are limits where also our science becomes more exciting, more questionable, and eventually incomprehensible. What physical science and life science have in common is, that studying the extremes is very instructive for learning what the general laws are. So we do experiments in, and theoretically analyze, the extremes.

Let me consider various possible extremes one by one, and disclose what was discovered about our physical world when subject to these extreme conditions.

\section{Extremely Fast}

According to the old laws of physics, any physical structure, regardless whether it is living or dead material, can be moved around with velocities that, in principle, can be arbitrarily large. This is not as evident as it may sound, since we can

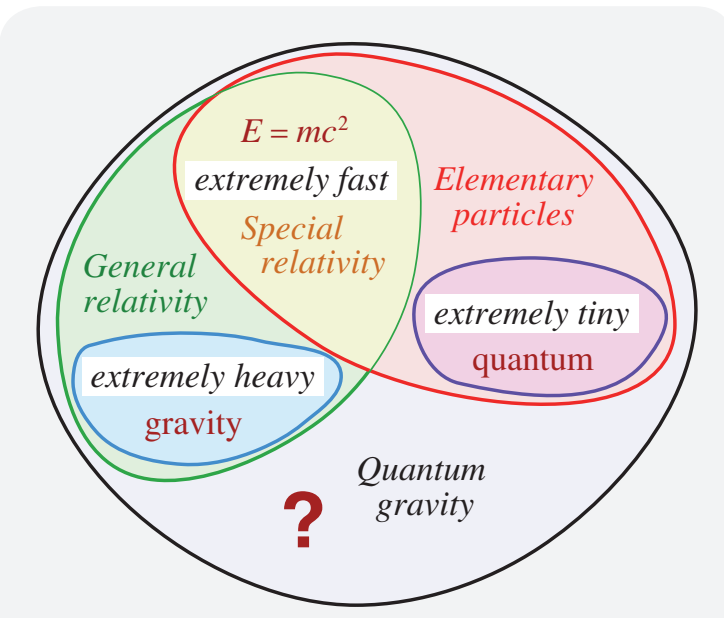

Figure 1. Extremes in physics. "Extremely fast" is described by the theory of Special Relativity; "extremely heavy" leads to Newton's gravity theory. Combining these two leads to General Relativity. "Extremely tiny" leads to quantum theory, and combining that with extremely fast gives us the theory of elementary particles. To combine all these extremes into one requires a theory for Quantum Gravity, which is not yet completely understood. imagine universes where such would not be the case - if you would try to move something, it would immediately break into pieces.

It was Albert Einstein who asked the question what an observer would see if (s)he moves as fast as the speed of light; when observing the surrounding universe, using light to observe things, you might, for instance, see nothing when you look behind you. Einstein's extremely ingenious discovery was, that one can imagine slight modifications of the laws of physics known at his time, in such a way that an observer would be able to look around and use light to observe things, exactly as if he were not moving at all, except for one limitation: (s)he cannot actually reach the speed of light regardless his/her efforts.

The speed of light is extremely large. In fractions of a microsecond light can move by hundreds of meters. This is why, in the older text books of physics, observers moving that fast were simply not considered. But since, today, most of our communications go with the speed of light, and contain not millions but billions of data per second, this domain that used to be extreme, has become very central in physics, ever since the beginning of the $20^{\text {th }}$ century.

The space we live in is three-dimensional. To locate any object, any point, in space, one needs exactly three coordinates, usually called $x, y$ and $z$. It was Hermann Minkowski who proposed to add time, $t$, as a new dimension, so that three dimensional space was replaced by a four dimensional space-time. Think of a train schedule, where it is indicated where the train is ( $x$ and $y$ together localize the train on the map, $z$ indicates how high it is above sea level), but also we add at what time, $t$, the train is supposed to be there.

When you change the velocity of something, this can be regarded as a rotation in Minkowski's space-time. In threespace, rotations are easy to visualize, and a physical law says that the internal properties of something do not change when you rotate it. This is a fundamental property of space. At first sight it seems not to be exactly valid: if I rotate a glass of water along a horizontal axis, the water may pour out. That, however, can be attributed to the presence of planet Earth below us, which possesses a gravitational field attracting the water. Similarly, changing the velocity of something can be regarded as a rotation in Minkowski space-time, and the fact that the internal properties of something, such as the question whether it is dead or alive, sick or healthy, do not change when you make such a rotation, is a fundamental property of Minkowski's space-time. Again, if the property seems to be not exactly valid, for instance if you feel more and more wind when you increase your speed, this can be attributed to the presence of air, which has a fixed velocity itself.

There are two different ways in which we can consider rotations: you can either rotate the object you are looking at, and compare what you see under the different conditions, or 
you can rotate the observer, that is, yourself, and look at the same object. In both cases you can ask whether the laws of physics themselves are affected by these rotations. In both cases, you modify the coordinates $x, y, z$, and $t$, by the rotated coordinates. Einstein found how one can formulate the laws such that they are guaranteed not to be affected by rotations, be it in three-space, or in Minkowski space-time.

The way things can rotate in space and time had already been formulated by my compatriot Hendrik Antoon Lorentz, in 1892 and 1895, well before Einstein (1905). There is something odd, however, about these rotations. Why is it that the speed of light is a limiting speed? The answer to this is that the rotations in Minkowski space-time are non Euclidean, so they differ from ordinary rotations. While in ordinary rotations the distance between two points always remains the same, rotations in Minkowski space are chosen to be such that the speed of light is always kept to be the same regardless by how much we rotate the coordinates.

A point on an object that rotates in ordinary space describes a circle. It can rotate around the circle as many times as one wishes to consider. If I would use different units of length for horizontal distances than for vertical distances, a point on an object that rotates along a horizontal axis would describe an ellipse - a flattened circle. Circles and ellipses are conic sections, but there exist also other conic sections: the parabola and the hyperbola. In Minkowski's space-time, a point on a rotating object may follow a hyperbola.

A hyperbola has two asymptotes; these are straight lines that are approached as closely as one wants by the hyperbola, but they are never touched. A wave of light, or a photon, follows the path on the asymptote; a rotated object cannot cross that asymptote, and this is why ordinary objects can never reach the speed of light - no matter how far one rotates it, the point has to stay on the hyperbola.

Lorentz had discovered that these are the rotations that leave the laws of electricity and magnetism unchanged. It was Einstein who made the next step: all laws of physics must stay unchanged when you consider these rotations, now called Lorentz rotations. And this does imply that Newton's laws of mechanics, the ones that were extremely successful in explaining the motion of planets and moons in elliptical orbits, had to be modified. At first sight, the modifications seemed to be minute, but the consequences for our physical world were gigantic.

In particular, it was found that whenever energy is added to a system, its mass will change: the mass change was found to be equal to the energy divided by the square of the speed of light, $c^{2}$. This change is minute, simply because $c^{2}$ is so big.

But it was already known that the atomic weights of the elements deviate slightly from what one would have expected if these atoms were composed of elementary particles, and these mass differences could be measured precisely. Now, Einstein realized that the mass changes were explained in his theory; they correspond to the energy by which the fundamental particles are bound together in the heavier atoms. If we call the observed mass defects $m$, then the associated energies must obey $E=m c^{2}$. It was thus deduced that the nuclei of the atoms of the elements in the periodic table contain huge amounts of energy. The astounding consequences of this observation changed the world.

In ordinary space, a particle is just a point. In Minkowsi space-time, we see the particles somewhere at every moment $t$ in time, and therefore, a particle is represented by a curve instead of a point.

At every given time $t$, the points in three-space form a section of Minkowski space-time, called the Cauchy surface. When we perform a Lorentz rotation, this Cauchy surface also rotates, it does not stay the same. This means that the question whether two events in Minkowski space are simultaneous, will be answered differently by observers with different velocities. Thus, the concept of simultaneity is relative, not absolute. This gives rise to many counter intuitive features in Einstein's relativity theory.

Since now we know that objects with any material substance in them, cannot surpass the velocity of light, we

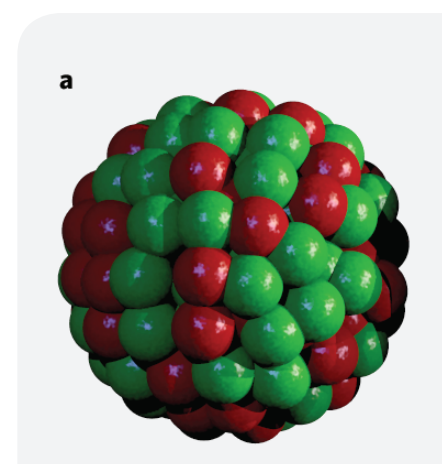

b

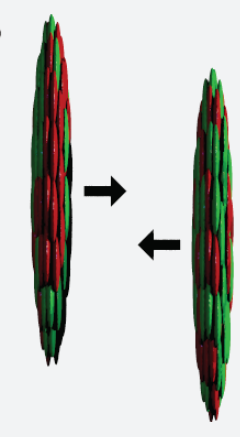

c

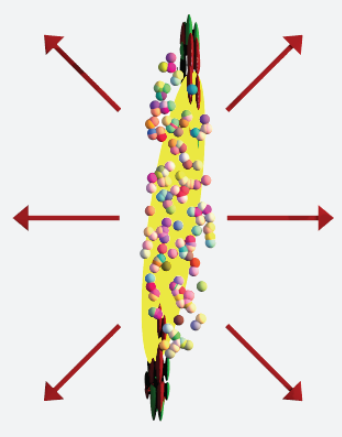

Figure 2. Heavy ion collision. In the Large Hadron Collider, nuclei of lead atoms (a) are accelerated to velocities so close to that of light that they are strongly contracted by Lorentz contraction. The actual contraction is even stronger than sketched in (b). When these collide (c), large numbers of particles are generated, occupying a tiny volume, so that, during a short period of time, the particle density is much greater than in the original nuclei. The exploding debris can now be studied. 
can now slightly modify our conception of extreme velocities: Velocities are extreme when they are extremely close to the speed of light. To reach such extreme velocities, extremely large amounts of kinetic energy have to be imparted to the object. What happens when such extremely energetic objects collide against one another has become a separate branch of physics: high-energy physics. The Large Hadron Collider, a particle physics laboratory near Geneva, Switzerland, is today's champion in reaching the most extreme velocities in this sense. Hadronic matter is accelerated there to more than $99.999999 \%$ of the speed of light. As of what happens at velocities even closer to the speed of light, there are still mysteries.

The theoretical doctrine needed to understand the extreme velocities is Einstein's theory of special relativity. There is however a more generalized relativity theory, General Relativity. About that, more later.

\section{Extremely Tiny}

Living organisms such as bacteria and viruses are tiny, but not at all as tiny as the tiniest structures physicists are studying. The discovery that extremely tiny objects (the sub-microscopic world) obey laws of physics that are fundamentally different from those of the macroscopic world (people, cars, planets) was made also at the beginning of the $20^{\text {th }}$ century. At that time, the strongest evidence for the existence of atoms and molecules came from the study of the laws of chemistry. In many respects, atoms and molecules behave very differently from larger and heavier objects, such as bacteria, let alone people and planets.

First of all, when two atoms are equal, they really are fundamentally equal. Any observable differences in behavior are irreproducible, or accountable to environment or other factors not under our control. When an experiment with beams of atoms or other fundamental particles is carried out, then, apart from purely statistical fluctuations, atoms of the same species are indistinguishable, and the outcomes of statistical experiments are completely reproducible. The doctrine required for the description of these objects is very different form classical mechanics, and it is summarized by just one word: Quantum.

Differently from relativity theory, Quantum Mechanics was developed by the basic insights of many scientists, such as Niels Bohr, Louis de Broglie, Werner Heisenberg, and Erwin Schrödinger among others. It also took much more time to understand it as we do now, and even now, it is much more difficult to explain.

The first thing physicists learned is that very tiny particles are associated to fields that form wave patterns. These waves contain all the information needed to describe a particle. Different species of particles carry - or are carried by - different kinds of oscillating fields. The frequencies of the oscillations are indicative for the energies these particles contain, while the wavelengths are linked to momentum, that is, velocity times mass. These observations may sound somewhat vague, but it was discovered by Erwin Schrödinger that the waves obey mathematical equations, wave equations, which turn out to replace the old Newtonian laws of mechanics: quantum mechanics. With these equations, quantum mechanics became a theory with high precision.

The amplitudes of the waves determine where the particles can most likely be found, and again these features were precisely laid down by mathematical equations. The equations tell us that the waves form certain patterns, and the ways these patterns move around tell us how particles move. It turns out that, if many, heavy particles are put together, the equations are such that they can be replaced by the older Newton equations, and these classical equations are much more accessible to intuitive understanding.

These discoveries came hand-in-hand with a much better understanding of the properties of atoms, beginning with Niels Bohr's crude model of the atom, and culminating into precision physics that allowed us to check and understand atoms fantastically precisely.

An atom consists of a very tiny nucleus, composed by what we call hadronic material, surrounded by electrons that orbit the nucleus at relatively larger distances. These electrons form wave patterns that become more and more complex as we consider larger and heavier atoms. The complexity comes from the fact that larger atoms contain many electrons. Carrying just one electron, the hydrogen atom is mostly spherically symmetric, but the 8 electrons orbiting oxygen nuclei form waves with four peaks, roughly taking the shape of a tetrahedron. When oxygen binds to hydrogen, the hydrogen nuclei are attracted by these peaks, and consequently, when two hydrogen atoms are bound to an oxygen atom, they do not sit opposite to each other, but form an angle of $105^{\circ}$. We are talking of a water molecule here, and its special shape gives water its unique properties. The description given here is very crude - the actual angular separation between two points of a tetrahedron is $109.5^{\circ}$, which makes clear that the tetrahedron does not explain everything; the behavior of the 10 electrons in a water molecule is very complex.

The tendency of carbon atoms to arrange themselves in long chains can be understood along similar lines. Quite generally, due to their wave patterns, electrons in atoms force other atoms to be attached under different angles, which give the resulting molecules their special shapes. These discoveries brought us closer to an understanding of the complex laws of chemistry that lie at the basis of life.

We reiterate that, by studying the extremes, physicists discover how laws of Nature may have to be reformulated to cope with the new situations. This will also be true for the other extremes that are to be discussed later. 
The physics of atoms is not at all at the limit of the very tiniest things we can study. The atomic nucleus is much smaller than the atom itself, and it is controlled by its own physical laws. These laws were at the center of theoretical and experimental physics around the mid $20^{\text {th }}$ century. By that time, it was understood that nuclei consist of protons and neutrons, particles of nearly equal mass, but totally different in their electromagnetic properties. The nucleus does not consist of protons and electrons, which had been the natural thing to suspect in earlier days. Today we know that the quantum mechanical laws controlling the electron do not allow it to occupy such small spaces as what we have inside a nucleus. The electron is far too light for that.

Protons and neutrons exert quite strong and complex forces on one another. By 1980, these forces were quite well understood qualitatively, but several decades were needed before accurate agreement was found between experimental measurements and theoretical calculations. We have even smaller building blocks, the quarks, of which there are 6 basic types. Three of these quarks are needed to form a hadronic particle such as the proton and the neutron. In addition, there are relatives of the electron, called leptons, of which we have also 6 types all taken together.

\section{Extremely Tiny and Extremely Fast}

To understand the laws of physics at scales as tiny as the atomic nucleus, we have to realize that all structures at such scales are generated by elementary particles having great velocities. This is the way it must be according to the laws of quantum mechanics. Thus, the physics of very tiny things on the one hand, and the laws of the greatest velocities on the other, merge when the extreme limits are concerned. And so it happens that the Large Hadron Collider, in its attempt to study the tiniest distance scales in physics, is designed also to produce particles with the highest possible velocities, thus combining two extremes in one.

Clearly, this limit now combines Einstein's special relativity theory with quantum mechanics. How to do this "quantum relativity" right, was a major problem during the $20^{\text {th }}$ century, but it was almost completely solved around the 1970s. The solution is called Quantum Field Theory. Many physicists contributed to this success. Paul Dirac discovered a relativistic equation for describing the wave function of an electron with relativistic velocities. He discovered that the equation works best if one allows for the presence of unlimited numbers of electrons, in addition to something completely new: positrons, which are the antiparticles of electrons. It was essential for this theory to work, that under certain conditions one can observe the successive creation of a particle and its annihilation shortly afterwards, but that this process becomes indistinguishable from the process where first an antiparticle is produced, after which that is annihilated again, where the points of creation and annihilation are interchanged.

Because of the quantum nature of these particles, one can sometimes not exactly tell whether its velocity was slower or faster than light, and even whether the particle went backwards in time. The crucial thing to keep in mind here was that the elementary field equations that describe all this, should obey the principle of causality: never allow any piece of information to be sent faster than light, but don't worry whether it was carried by a particle or an antiparticle.

The theory that ensued was based on the notion of fields, to which quantum equations were imposed, hence the name quantum field theory. It eventually evolved into a rigorous scheme, forcing the behavior of all known elementary particles to be described by a concise set of equations: the Standard Model. All known particles now carry three types of charges: first we have the electromagnetic charges, which were already known and understood since James Clark Maxwell wrote down his famous equations around 1861. Since Dirac's work, we know how the electromagnetic force acts upon the tiniest elementary particles. Secondly, we have the weak charges. The weak force caused by these charges actually mixes with the electromagnetic force, which is why today we speak of the electroweak forces.

Finally, there is the strong force. This force only acts on what we call hadronic particles. In contrast to the electric charges, which are simply either positive or negative, the strong charges come in three types, for which we use the analogy with color: red, green and blue. The antiquarks have the conjugated colored charges: cyan, magenta and yellow. These forces are so strong that their action can be very nonlinear, which makes the calculations concerning the strong force extremely complicated: during the short period of a collision of particles, the strong force can act many times. The forces by which these charges act on one another are a generalization of the concept of electromagnetic force, called the Yang-Mills force. While electromagnetic forces are carried along by a single type of particle, the photon, the other forces are carried by three kinds of weak photons, called $\mathrm{W}^{+}, \mathrm{W}^{-}$and $Z^{0}$, and eight types of strong photons, the so-called gluons. While the photon was a neutral particle, the gluons do carry color charges themselves.

There is one very special force in nature that is not, or not yet, captured by our understanding of the Standard Model, which is the gravitational force. Under normal circumstances, the gravitational force is so weak that is hardly affects our understanding of the physical laws concerning particles of matter. The hadronic particles circulating in the Large Hadron Collider do not feel Earth's gravity simply because they are moving much too fast for that.

However, when we penetrate the world of the tiny things deeper, we need to look at particles with ever increasing 
amounts of energy, because that makes their waves tinier. Gravity couples to mass, and mass is energy, as Einstein had discovered, and this is why the gravitational force slowly but steadily gains in strength when we continue our journey into the things of the small. Eventually, gravity will overtake all other kinds of forces. When this happens, we approach a new extreme limit. We return to that.

\section{Extremely Heavy}

When objects possess a large amount of mass, they tend to become heavy. This is a direct consequence of the fact that the gravitational forces only depend on mass and nothing else. When we study heavy objects, we are eventually led to heavy objects in outer space: planets and stars. These objects are sensitive to their own gravitational fields, and as soon as they reach a certain limiting amount of mass, they attain the only allowable stable shape, that of a sphere - possibly flattened to some extent because of rotation.

These heavy objects are surrounded by a gravitational field, which accelerate other, nearby objects. As long as these gravitational fields are not too strong, they present little problems when we wish to describe them in terms of physical equations. As was discovered by Johannes Kepler, and explained by Isaac Newton, the orbits of stars and planets in the gravitational fields of others, take the shape of ellipses.

\section{Extremely Dense}

When the gravitational field reaches certain thresholds, particles inside a gravitating body may undergo phase transitions into forms of matter we still know fairly little about. Such a thing happens in a "neutron star". Here, the original atoms are compressed to the extent that protons and neutrons hardly fit in the space they have. Most of the electrons do not fit there at all and they are forced to move out. This in turn causes most of the protons to absorb electrons and turn into neutrons. Thus, mainly neutrons remain inside. This results into what we call a neutron star. Neutron stars are observed because they tend to rotate very fast, hundreds of rotations per second. All radio emissions fluctuate in accordance, making them easy to recognize as pulsating sources of radio signals, or pulsars.

To illustrate how extreme these densities are, we note that our sun would turn into a neutron star only if we could compress it such that its diameter is a few tens of kilometers. The actual diameter of the sun is over a million kilometers.

Other features also tend to become extreme in the insides of a neutron star. Its magnetic field, for instance can become hundreds of millions times stronger than fields produced in our laboratories. If a pulsar would approach us as close as where the moon is now, our watches would stop running. The emitted radiation would be lethal for us at that distance. One pulsar has a rotation rate clocked to be over 700 revolutions per second.
Even higher densities can be considered. In the Large Hadron Collider, nuclei of lead atoms, with all 82 electrons stripped off, can be made to collide against one another. During the collision, for a short time interval, the protons and neutrons of these nuclei are forced into a space that is even smaller than inside a neutron star. Also the temperatures are the highest that can be obtained in a laboratory on Earth.

During the earliest phases of the universe's existence, the universe expanded very rapidly, and it all began when all particles constituting the presently observable part of the universe, were compressed into a single point. Up to times less than a microsecond after the beginning, the conditions were much more extreme than in any laboratory on Earth. Also the temperature was much higher than what we can study in our machines. To some extent, one can calculate how matter must have behaved, but when densities and temperatures were much higher, phenomena occurred that we can only speculate about. However, we know what our universe looks like today, and from that we can infer at least some properties of matter during the initial phases, since these must have been the reason why the universe expanded very rapidly and homogeneously to become what it is now. We shortly return to the beginning of the universe later.

\section{Extremely Heavy and Extremely Fast}

Once again, all changes when, due to the action of very strong gravitational fields, objects moving near it, and sometimes also the material out of which the source object is made itself, attain velocities nearing that of light. What we then see is relativistic gravity. Here, mass and energy get intertwined, and the energy residing in the gravitational field itself can no longer be ignored. Very precise measurements in ingenuous experiments have revealed that gravity always acts on mass and nothing else. This gives us severe restrictions when designed a theory for relativistic gravity. The solution was found by Albert Einstein in 1915: General Relativity. His theory combines extremely heavy objects with extremely high velocities.

To understand what happens, our picture of space and time has to be modified once more. Since high velocities amount to rotations in Minkowski space-time, one finds that such rotations are generated near a heavy gravitating body. This causes all sorts of deformations in space-time, and the net result of this was that Einstein was forced to contemplate curvature in space and time.

The most conspicuous effect of this curvature is due to the curvature in the time direction: inside the gravitational potential well of a gravitating body not only all clocks seem to proceed slower than at farther distances, but also all other physical processes appear to slow down. A local observer will not directly notice this, because his own body and all his measuring devices slow down as well, so we are talking of a property of space and time themselves. 
Also in the purely space-like direction one finds curvature to occur. Distances between points in space deep inside a gravitational potential, appear to be greater than if we used a flat coordinate frame and counted the lines in between.

It all becomes a coherent theory only if we add the equations, but these tend to be rather complicated, since curvature depends on direction, in a very intricate way. It so happened that Einstein did not have much choice. Only one theory was possible, with the exception of slight, unknown modifications, possible only at the extremely large scale given by the size of the universe itself.

Thus, the solution as to what happens in this extreme case was found, and it was basically unique. General relativity came with various possible ways to test it. Direct conflicts with observations were never encountered, although we do notice gravitational fields inside and around galaxies that are difficult to account for, if they are assumed to be due to matter at all. The missing source of these gravitational fields is called "dark matter", but the possibility that General Relativity itself requires amendments is seriously studied by quite a few researchers.

"Extremely heavy and extremely fast" has an inescapable limit. General Relativity allows us to compute precisely what happens when the gravitational force of a heavy object surpasses a magic boundary point: particles can be gravitated towards the speed of light, and at first sight nothing may seem to stop them from continuing their acceleration beyond that. What happens then, however, is that space-time curvature reaches a turning point. The equations are quite clear about this: the gravitating body then collapses entirely, and it turns into what is known by the name "black hole".

Since its equations are known and understood, we know quite a lot about black holes. They are extremely violent. They are surrounded by a mathematically defined surface called the horizon. In principle, one can ask what an observer will see who falls through this horizon. Besides experiencing queer gravitational forces acting on his body, the observer can, in principle, survive the journey through the horizon, but very quickly this observer will end up in a kind of apocalypse that no-one can survive. Only if the original black hole was deformed by rotation, there may till be another universe waiting ahead of our observer, but contact with our own universe will have terminated.

Black holes are black, nothing will come out of them, unless we combine the extremely heavy and the extremely fast, with the physics of the extremely tiny distances. There, quantum mechanics tells us a different story.

\section{Extremely Heavy, Extremely Fast, and Extremely Tiny}

This is the one extreme limit where not only strange things happen, but we are really extremely ignorant about the exact way to describe it. What we are fairly certain about, is a theoretical discovery made by Stephen Hawking in the 1970s. He found that a new prediction can be deduced by applying the laws of quantized fields to the curved space and time of a black hole. Even if an observer falling into a black hole would describe his environment as being completely void of any elementary particles, the outside observer will detect particles coming out. The reason for this is that the outside observer experiences time in a way that is fundamentally different from what observers see who fall in: for the outside observer, time at the horizon will seem to come to a halt, while someone going in will observe that time continues. Time is mathematically linked to the notion of energy, and energy is used to define what a particle really is: an object carrying a certain amount of energy. Consequently, observers outside a black hole will observe particles even where observers falling in see none.

Important as this discovery is, it does not exactly yield the complete set of equations needed for a precise description of the world in this extreme limit. Candidate theories that might do the job have been proposed. Some theories posit that gravitational forces might eventually become independent of scale, so that, in this limit, we simply approach an asymptote, where no more things of interest will happen; this theory is called "gravitational safety". Another theory takes it that space and time can be partitioned into small domains, connected by lines and closed loops; this theory is called "Ioop quantum gravity". The most powerful and imaginative approach is collectively called "superstring theory". This theory comes in various versions, such as "M-theory", where the strings are replaced by giant matrices. However, the promises of all these theories are often exaggerated by their proponents. Fact is that, we still do not know or understand the resulting equations very well.

\section{Extremely Tiny and Extremely Big}

There are other interesting extremes. We may consider very big quantities of very tiny particles. This could enable us to consider theories for the entire universe. The universe is old, some 13.8 billion years. In the beginning of the $20^{\text {th }}$ century it was found, by Edwin Hubble, that the universe is rapidly expanding. This means that, in the far past, the universe must have been a lot smaller than at present, and all evidence is pointing towards the idea that the entire part of the universe that can presently be seen in our telescopes, may have originated in a single point.

The history of the universe has been reconstructed precisely in recent years. Saul Perlmutter, Brian Schmidt and Adam Riess received the 2011 Nobel Prize for their work in determining the expansion rate of the universe. For this they had used observations of supernova explosions of stars. A supernova can also be considered as the most extreme events that can take place inside a star, destroying most of it. Supernovae can be used as standard candles to shine more light on 
the exact structure the universe took from the very first times onwards. Surprisingly, they found that the universe expands by an accelerating pace. Before this acceleration effect was known, there were some discrepancies in the determination of the universe's age. Now, these discrepancies have been removed, but the acceleration was left to be explained. Most likely, the cause is a term in Einstein's General Relativity theory that is not very well understood: the cosmological constant. This constant was usually assumed to be zero, but now it is realized that this cannot be so. This constant is one of the smallest non-vanishing constants we have in describing the physical world, and we do not know why it is there.

As stated before, in earlier epochs, the universe must have gone through a phase where it was extremely dense. It must also have been extremely hot, since expansion goes with cooling down. Careful observations and theoretical deduction have revealed that the initial expansion rate must have been enormous; the universe must have been expanding in an exponential fashion. This would be needed if indeed everything originated in a point, which in turn is a much favored picture just because the early universe was observed to have been extremely homogeneous. Even the most distant extents of the universe must all share a common ancestor point in their pasts.

We have the equations required for an exponentially expanding universe. This "inflationary universe" results if we assume that matter in the distant past were in a more symmetric phase than what it is in at present. The theory agrees with the minute fluctuations observed in radiation originating from these early phases our universe has been in. This subject has presently evolved into an independent branch of science, called cosmology. Less than half a century ago, speculations on this topic could be dismissed as "science fiction". Today, it still contains fiction, but the scientific part of this doctrine has matured immensely.

\section{Extremely Cold}

At low temperatures, particles of matter tend to move very slowly. While at ordinary temperatures most properties of matter can be understood using ordinary, Newtonian mechanics, very low temperatures require quantum mechanics to understand what happens. These features can indeed be exotic. At the beginning of the $20^{\text {th }}$ century, Dutch physicist Heike Kamerlingh Onnes decided to study the properties of atoms by investigating metals at the lowest temperature he could achieve, which was of the order of one degree above absolute zero. When checking the electric conductance of mercury, which is frozen solid at these temperatures, he found something totally unexpected: all resistance against electric currents disappears, or, mercury becomes superconducting.

At ordinary pressures, all substances except helium become solid at sufficiently low temperatures. Besides mercury, many other materials were found to become superconducting. This is a quantum effect, only understood much later. In helium, the isotope ${ }^{4} \mathrm{He}$ becomes a superfluid: all resistance against flow disappears. When this material is pumped through a pipe, it shows no resistance at all (as long as the velocities are not to great). This is also a quantum mechanical effect, similar to superconductivity. Kamerlingh Onnes had encountered superfluidity of helium as well, as was later deduced from the notes he made, but he had not interpreted what he saw the way we do now.

Today, there still is a lot of interest in low temperature physics. Individual atoms, forced to sit in certain patterns, are now cooled to less than a millionth of one degree Kelvin. Many exotic phenomena take place in these extreme circumstances.

\section{Extremely Complex}

Finally, our universe is extremely big, consisting of gigantic amounts of particles, and consequently these particles must be behaving together in a highly complex manner. Needless to say, only extremely complex phenomena can be held responsible for many features occurring in our part of the universe. Without doubt, the most complex feature of all will have been the emergence and evolution of life on at least one planet. So we return to the life sciences, where this talk also began.

Only recently a long held suspicion has been confirmed: there exist enormous numbers of planets near the stars surrounding our Sun. Many of these planets are very different from planet Earth, but also gigantic numbers of planets must exist that are very similar to Earth. Are these also teeming with life, or is our planet a single, lonely exception having living creatures accidently colonizing it, changing its surface completely?

Complexity raises several other mysteries concerning our universe. As the reader may have noticed, combining several extremes together leads to situations that are even more difficult to comprehend. When combining extremely heavy with extremely fast and extremely tiny, we approach the domain of quantum gravity, which is still very poorly understood. There may be a good reason for this. All extremes converge towards this point, and so it might be that only a complete theory for all physical forces, all elementary particles, and all fundamental principles together may be required to get this domain of nature properly mapped. At the same time, one may notice that then one has a description of the entire universe. This goes by the nickname "Theory of Everything". But a theory that is able to account for everything that is happening in the entire universe may well be required to possess seeds of all sources of complexity in this universe. That may be quite something to demand of a single theory. Such considerations are reasons to doubt whether ideas such as 
"superstring theory" contain the right mix of super-complex behavior. This speaker suspects that a lot more will be needed. I advocate procedures that are much more precise than what is presently in use. Perhaps as much as the combined intellect of all of humanity is what is needed to discover all the truths about nature in the ultimate extreme case. Individually, we are too stupid.

Complexity may well be the hardest condition in the physical world to study and comprehend. What I wished to illustrate in this talk is that most physical phenomena and laws can be pretty well understood and analyzed. Only when the circumstances become extreme, new challenges arise. I named a number of such challenges, and indicated how investigators cope with them. While they do, they will without doubt encounter new extreme conditions and new challenges that keep us all sharp. Eventually, there is reason to hope that, one day, we can combine all extremes to fit in one universal theoretical description of nature. 